\title{
Efectos de la sal de estradiol y de la duración del tratamiento con progesterona sobre el porcentaje de preñez a la IATF
}

\author{
Biondini, M. ; Zangrilli, G. ${ }^{2}$; Preisseger, G. ${ }^{2}$; Callejas, S. ${ }^{1}$ \\ ${ }^{1}$ Área de Reproducción, Facultad de Ciencias Veterinarias, Universidad Nacional del Centro de la Provincia de \\ Buenos Aires, Paraje Arroyo Seco s/n, Tandil (7000), Buenos Aires, Argentina. Tel.: 02293-439850. \\ E-mail: sscallejas@gmail.com. \\ ${ }^{2}$ Actividad Privada. Tandil, Buenos Aires, Argentina.
}

\begin{abstract}
Resumen
Biondini, M.; Zangrilli, G.; Preisseger, G.; Callejas, S.: Efectos de la sal de estradiol y de la duración del tratamiento con progesterona sobre el porcentaje de preñez a la IATF. Rev. vet. 22: 2, 127-130, 2011. Se utilizaron 629 vaquillonas Angus (13-15 meses), pertenecientes a dos establecimientos (EI: 376, EII: 253) para evaluar el efecto de dos tratamientos para sincronizar la ovulación (cipionato-CPE vs benzoato de estradiol-BE) utilizando dispositivos con progesterona (DISP) y evaluando la permanencia del DISP (7 vs 8 días) sobre el porcentaje de preñez a la IATF. En cada establecimiento se formaron dos grupos. Grupo CPE: el día 0, se colocó un DISP (EI: 1 g; EII: 0,558 g de progesterona) $+2 \mathrm{mg} \mathrm{BE}$. El día 7 u 8 se retiró el DISP, se administró $0,150 \mathrm{mg}$ de D-Cloprostenol y CPE. Grupo BE: ídem tratamiento anterior utilizando BE $24 \mathrm{~h}$ post DISP sustituyendo al CPE. El servicio se realizó por IATF (4 toros en EI y 2 toros en EII). El día de retiro del DISP las vaquillonas del EI fueron pintadas en la base de la cola. Al realizar la IATF (51-52 h post DISP), las vaquillonas pintadas de EI y todas las del EII recibieron 0,0126 mg de acetato de Buserelina. EI: En los animales despintados la interacción sal de estradiol x toro fue significativa $(\mathrm{p}=0,02)$. Con uno de los toros se observó menor preñez al usar CPE $(53,3 \%$ vs $85,4 \%, \mathrm{p}<0,05$ ), no así en los otros toros. La permanencia del DISP no afectó la preñez ( 7 días: $66,5 \%$ y 8 días: $64,2 \% ; p>0,05)$. En los animales pintados $(56,5 \%)$ y en el EII $(52,9 \%)$ no hubo efecto de la sal de estradiol, permanencia del DISP, toro e interacciones $(p>0,05)$. Se concluye que, en las condiciones del presente trabajo, el uso de CPE en lugar del BE permite obtener en la mayoría de las veces similares porcentajes de preñez; no obstante, en algún caso la preñez puede afectarse negativamente. La duración del tratamiento ( 7 u 8 días) no afecta la preñez.
\end{abstract}

Palabras clave: vaquillonas, cipionato de estradiol, IATF, preñez.

\begin{abstract}
Biondini, M.; Zangrilli, G.; Preisseger, G.; Callejas, S.: Effects of estradiol salt and duration of progesterone treatment upon pregnancy rate after FTAI. Rev. vet. 22: 2, 127-130, 2011. Six hundred and twenty-nine Angus heifers (13-15 month-old), located in two farms (FI: 376 and FII: 253) were used to evaluate the effect of two treatments for ovulation synchronization using intravaginal progesterone device (IVD) with estradiol cypionate (ECP) or benzoate (EB) and evaluating IVD permanence (7 vs. 8 days) on the pregnancy rate of FTAI. Heifers were assigned into two groups in each farm. ECP Group: day 0, insertion of an IVD (EI: $1 \mathrm{~g}$; EII: 0.558 $\mathrm{g}$ of progesterone) $+\mathrm{EB}$. On day 7 or 8, IVD was removed and D-cloprostenol and ECP were injected. EB Group: similar to previous treatment but using EB $24 \mathrm{~h}$ post IVD instead of ECP. Breeding was performed by FTAI using 4 bulls in FI and 2 bulls in FII. The day of removal of devices heifers in FI were tail painted. At FTAI (51-52 h post IVD), heifers painted in FI and all of the FII received $0.0126 \mathrm{mg}$ of Busereline acetate. FI: for faded paint animals, interaction of estradiol salt per bull was significant $(\mathrm{p}=0.02)$. With one of the bulls, a low pregnancy rate was observed using ECP $(53.3 \%$ vs $85.4 \%, \mathrm{p}<0.05)$ but not in the other bulls. Treatment duration did not affect the pregnancy rate ( 7 days: $66.5 \%$ and 8 days: $64.2 \%$; $p>0.05$ ). Estradiol salts, treatment duration, bulls and interaction effects on pregnancy rate were not observed either in painted (56.5\%) or FII (52.9\%) animals. In conclusion, under work conditions, the use of ECP instead of EB can produce, in most of the cases similar pregnancy rates; however, in some cases, it may cause a negative effect. The duration of treatment ( 7 or 8 days) does not affect pregnancy rate.
\end{abstract}

Key words: heifers, estradiol cypionate, FTAI, pregnancy. 


\section{INTRODUCCIÓN}

El uso de dispositivos intravaginales con progesterona combinado con benzoato de estradiol, administrado al momento de colocarlo y 24 horas luego de retirar el mismo, sumado a una inyección de un agente luteolítico al final del tratamiento, ha sido utilizado para controlar el ciclo estral e implementar programas de inseminación artificial a tiempo fijo (IATF) ${ }^{6}$. Este tratamiento permite sincronizar la ovulación de los animales que se encuentran ciclando, así como inducir actividad en aquéllos que están en anestro.

En vaquillonas que entran al servicio y son incorporadas a un programa de IATF, se ha utilizado el protocolo de control del ciclo estral mencionado precedentemente con resultados satisfactorios, con un rango de preñez de 45 a $80 \%^{2,6,8}$.

Con la finalidad de simplificar los protocolos de sincronización de la ovulación se han realizado trabajos que han sustituido el uso del benzoato de estradiol por cipionato de estradiol (CPE) inyectándolo en el momento de retirar el dispositivo intravaginal ${ }^{5}$ o $\mathrm{GnRH}$ administrada en el momento de realizar la IATF $^{3}$. De esta manera se simplifica el tratamiento al reducir de 4 a 3 , el número de veces que los animales pasan por la manga.

Si bien hay trabajos que han mostrado que se obtienen resultados similares de preñez entre las dos sales de estradiol (cipionato inyectado al retirar el dispositivo o benzoato a las 24 horas posteriores), surge de interés aumentar la casuística y evaluar si esta respuesta es independiente de la duración del tratamiento. En consecuencia, el objetivo del presente trabajo fue evaluar el efecto de la sal de estradiol utilizada (cipionato vs. benzoato) y de la duración del tratamiento ( 7 vs. 8 días) sobre el porcentaje de preñez a la IATF en vaquillonas con 15 meses de edad.

\section{MATERIAL Y MÉTODOS}

Lugar, animales, alimentación. El trabajo se realizó en dos establecimientos comerciales (EI y EII) ubicados en el partido de Azul (EI) y en la localidad Tedin Uriburu, partido de Benito Juárez (EII), ambos en la Provincia de Buenos Aires, Argentina. Se utilizaron 629 vaquillonas Angus (EI: 376, EII: 253) con 13 a 15 meses de edad y un peso que varió de 285 a $315 \mathrm{~kg}$. La alimentación en ambos establecimientos se basó en suplementación con silo de maíz, maíz en grano, expeler de girasol y un núcleo vitamínico. Este régimen alimenticio comenzó 4 meses antes de iniciar el tratamiento de sincronización de celos.

Tratamientos y servicio. Dentro de cada establecimiento las vaquillonas fueron distribuidas al azar a dos grupos que recibieron los siguientes tratamientos:

1. Grupo CPE: el día 0, se colocó un dispositivo intravaginal con $1 \mathrm{~g}$ de progesterona (Cronipres tres usos, Biogénesis Bagó) en el EI y 0,558 g de proges- terona en el EII (Cronipres M15, Biogénesis Bagó) más una inyección de $2 \mathrm{mg}$ de benzoato de estradiol (Bioestrogen, Biogénesis Bagó). El día 7 (CPE 7) u 8 (CPE 8) se retiró el dispositivo, se administró 0,150 mg de D-Cloprostenol (Enzaprost D-C, Biogénesis Bagó) y 1 mg CPE (ECP, Lab. Konig).

2. Grupo BE: Ídem tratamiento anterior con la salvedad que se administró $1 \mathrm{mg}$ de benzoato de estradiol a las 24 horas de retirado el dispositivo en lugar del CPE.

El servicio se realizó por IATF (51-52 horas de retirado el dispositivo), utilizando semen congelado/ descongelado, en pajuelas de $0,5 \mathrm{ml}$, provenientes de 4 toros en el EI (A, B, C y D) y de 2 toros en el EII (A y D). Las partidas de semen fueron analizadas en el laboratorio del Área de Reproducción de la Facultad de Ciencias Veterinarias, Universidad Nacional del Centro de la Provincia de Buenos Aires, indicando que eran aptas para ser utilizadas en inseminación (Tabla 1).

El día de retiro del dispositivo las vaquillonas del EI fueron pintadas en la base de la cola. En el momento de realizar la IATF, aquellas vaquillonas que estuvieron pintadas recibieron una inyección de $0,0126 \mathrm{mg}$ de acetato de Buserelina (Gonaxal, Biogénesis Bagó); en el EII, todas las vaquillonas fueron inyectadas con dicha droga.

Diagnóstico de gestación. Se realizó por medio de ultrasonografía a los 30 días posteriores a la IATF (transductor transrrectal lineal de $5 \mathrm{MHz}$, Chison D 600 VET, China).

Análisis estadístico. Se evaluaron los efectos de la sal de estradiol (CPE vs BE), de la duración del tratamiento (7 vs 8 días), del toro y sus interacciones sobre el porcentaje de preñez a la IATF. Se utilizó el PROC CATMOD del paquete estadístico SAS ${ }^{12}$ fijándose un nivel de confianza del $95 \%(\alpha=0,05)$

\section{RESULTADOS}

Establecimiento 1. El $16,5 \%$ de las vaquillonas se encontraron pintadas en el momento de realizar la IATF, no difiriendo entre tratamientos, duración del tratamiento, toro utilizado o sus interacciones. En los animales despintados se observó un efecto significativo de la interacción de la sal de estradiol $\mathrm{x}$ toro $(\mathrm{p}=0,02)$. 
Tabla 2. Porcentajes de preñez en vaquillonas IATF según el toro y la sal de estradiol utilizada.

\begin{tabular}{cclc}
\hline toros & $\mathrm{E}_{2}$ & \multicolumn{2}{c}{ preñez $(\%) \mathrm{y} \mathrm{N}^{\mathrm{o}}$ de animales } \\
\hline \multirow{2}{*}{$\mathrm{A}$} & $\mathrm{CPE}$ & $58,1(18 / 31)$ & $61,7(37 / 60)$ \\
& $\mathrm{BE}$ & $65,5(19 / 29)$ & \\
$\mathrm{B}$ & $\mathrm{CPE}$ & $53,3^{\mathrm{a}}(24 / 45)$ & $68,6(59 / 86)$ \\
& $\mathrm{BE}$ & $85,4^{\mathrm{b}}(35 / 41)$ & \\
$\mathrm{C}$ & $\mathrm{CPE}$ & $63,6(28 / 44)$ & $60,0(51 / 85)$ \\
& $\mathrm{BE}$ & $56,1(23 / 41)$ & \\
$\mathrm{D}$ & $\mathrm{CPE}$ & $88,1(37 / 42)$ & $80,7(67 / 83)$ \\
\hline
\end{tabular}

$\mathrm{E}_{2}$ : sal de estradiol, CPE: cipionato, BE: benzoato, ${ }^{\mathrm{a}, \mathrm{b}}$ valores con superíndices diferentes difieren: $\mathrm{p}<0,01$.

Tabla 3. Porcentajes de preñez en vaquillonas sincronizadas con un dispositivo intravaginal con progesterona, según la sal de estradiol utilizada, la duración del tratamiento y el toro.

\begin{tabular}{ccc}
\hline efectos principales & preñez $(\%)$ \\
\hline CPE & sal de estradiol \\
BE & & $52,2(120 / 230)$ \\
7 días & duración & $53,4(174 / 326)$ \\
8 días & $49,1(139 / 283)$ \\
A & toros & $53,5(159 / 297)$ \\
D & & $53,7(245 / 456)$ \\
\hline
\end{tabular}

Sal de estradiol: CPE: cipionato; BE: benzoato.

Las vaquillonas que fueron inseminadas con el toro B se preñaron en menor proporción cuando recibieron CPE para sincronizar la ovulación, no observándose diferencias significativa entre sales de estradiol al utilizar los otros toros (Tabla 2).

El tiempo que permaneció colocado el dispositivo no afectó el porcentaje de preñez ( 7 días: $66,5 \%$ y 8 días: $64,2 \% ; p>0,05)$. En los animales pintados no se observaron efectos del tiempo en que permaneció colocado el dispositivo, de la sal de estradiol utilizada para sincronizar la ovulación, del toro ni de sus interacciones $(\mathrm{p}>0,05 ; 56,5 \%)$.

Establecimiento 2. El porcentaje de preñez $(52,9 \%)$ no difirió entre la sal de estradiol, duración del tratamiento y toros utilizados (Tabla 3, $p>0,05$ ). Las interacciones tampoco fueron significativas $(p>0,05)$.

\section{DISCUSIÓN}

Se han realizado trabajos en los que no se han observado diferencias significativas en el porcentaje de preñez al utilizar CPE administrado en el momento de retirar el dispositivo intravaginal con progesterona comparado con la inyección de BE 24 horas después (en vacas con cría ${ }^{7,10}$ y vaquillonas ${ }^{11}$ ); lo cual es coincidente con la mayor parte de los resultados obtenidos en el presente estudio. No obstante, se observó que con uno de los toros utilizados el porcentaje de preñez fue menor al emplear CPE. Si bien los análisis que se hicieron en el laboratorio indicaron que todos los toros eran aptos para ser utilizado en IATF, se podría hipotetizar que existen uno o más factores que no serían determinados en el análisis y que influirían en la fertilidad.

Trabajos realizados en el Área de Reproducción de la Facultad de Ciencias Veterinarias de la UNICEN ${ }^{1}$, han mostrado que cuando se inyecta CPE en el momento de retirar el dispositivo intravaginal con progesterona, se produce una mayor dispersión de la ovulación (CPE: 54 a 78 h vs BE: 66 h). Este hecho permitiría hipotetizar que podrían existir diferencias entre toros en la capacidad de los espermatozoides para mantener en el tiempo su aptitud para fecundar y/o dar un embrión viable. Esto podría haber ocurrido con el toro B, en el establecimiento 1, en el cual se observó que cuando se usó el CPE se obtuvo un menor porcentaje de preñez; no así con el $\mathrm{BE}$ en donde al concentrarse las ovulaciones, las exigencias del semen podrían ser menores. Futuros trabajos deberán abordar este aspecto, el cual resulta de suma importancia ya que permitiría definir el protocolo más adecuado de control del ciclo estral, según el tipo de toro.

Con respecto a la duración del tratamiento, el tiempo de permanencia del dispositivo en la vagina ( 7 u 8 días) fue igualmente efectivo, coincidiendo con trabajos que fueron realizados en vaquillonas ${ }^{4}$. Los porcentajes de preñez observados en los dos establecimientos (1: $64,9 \%$ y $2: 52,9 \%$ ) resultaron comprendidos en el rango citado en la bibliografía para vaquillonas con 15 meses de edad, que varía del 44 al $80 \%^{8,9}$.

Se concluye que, en las condiciones del presente trabajo, el uso de CPE administrado en el momento de retirar el dispositivo intravaginal con progesterona en lugar del BE permite obtener en la mayoría de las veces similares porcentajes de preñez. No obstante, ésta puede afectarse negativamente en algún caso, lo cual dependería del toro utilizado. Por otro lado, los dispositivos pueden permanecer colocados durante 7 u 8 días sin afectar la eficiencia reproductiva. Futuros trabajos deberán clarificar la relación que pueda existir entre el toro utilizado en la IATF, el uso del CPE inyectado al retirar el dispositivo intravaginal con progesterona y la respuesta reproductiva

\section{REFERENCIAS}

1. Callejas S, Uslenghi G, Larghi J, Cledou G, Cabodevila J. 2011. Uso del cipionato de estradiol para sincronizar ovulaciones en vaquillonas Holando. Rev Arg Prod Anim 31 (Supl 1): 409.

2. Bó G, Chesta P, Cutaia L. 2008. Claves para una IATF exitosa en rodeos de cría, Anales Cuartas Jornadas Taurus de Reproducción Bovina, Pilar, Buenos Aires (Argentina), p 14-33.

3. Cesaroni G, Butler H, Mc Dermott E, Cano A. 2000. Preñez de vaquillonas inseminadas a tiempo fijo después de un tratamiento con CIDR asociado con GnRH o con 
benzoato de estradiol aplicado 0 o 24 hs postratamiento. Rev Taurus 6: 20-25.

4. Chesta P, Cuataia L, Bó G. 2003. Efecto del tratamiento con DIB por 7 u 8 días en los porcentajes de preñez en vaquillonas cruza cebú inseminadas a tiempo fijo, Anales $V$ Simposio Internacional de Reproducción Animal, Córdoba (Argentina), p. 387.

5. Colazo M, Martinez M, Whittaker P, Kastelic J, Mapletoft, R. 2002. Estradiol cypionate (ECP) in CIDR-B based programs for fixed-time AI in beef heifers. Theriogenology 57: 371.

6. Colazo M, Kastelic P, Whittaker P., Gavaga Q, Wilde R, Mapletoft R. 2004. Fertility in beef cattle given a new or previously used CIDR insert and estradiol, with or without progesterone. Anim Reprod Sci 81: 25-34.

7. Giacusa N, Cutaia L, Bó G. 2005. Efecto de la utilización de cipionato de estradiol como inductor de la ovulación aplicado al momento del retiro de un dispositivo con P4 o 24 h más tarde sobre los porcentajes de preñez en vacas con cría, Anales VI Simposio Internacional de Reproducción Animal, Córdoba (Argentina), p. 404.

8. Lares S, Fernández-Francia G, Formia N, Giovaninni R, Massara N, de la Sota R.L. 2005. Eficacia de la utilización de un dispositivo intravaginal con progesterona monouso sobre la tasa de preñez en vaquillonas para carne de 15 meses, Anales VI Simposio Internacional de Reproducción Animal, Córdoba (Argentina), p. 405.
9. Marcantonio S, Polito J, Péndola C, Paramidani E, Udaquiola M, Capdevielle E, Spinetta E, Confalonieri J, Casaretto C, Gambarotta, M. 2011. Resultados de campo de programas de IATF en base a progesterona, sales de estradiol y prostaglandina obtenidos en 17 rodeos de cría durante 2001-2009, Anales VII Simposio Internacional de Reproducción Animal, Córdoba (Argentina), p. 352.

10. Rocha D, Bescow A, Azeredo D, Ponsati D, Kuhl F, Mattos R, Gregory R. 2005. Efecto del uso de distintos ésteres de estradiol como inductor de ovulación sobre la tasa de preñez en programas de inseminación artificial a tiempo fijo, Anales VI Simposio Internacional de Reproducción Animal, Córdoba (Argentina), p. 419.

11. Stahringer R, Vispo P. 2005. Efecto de la administración de dos sales de estrógenos y del momento de su aplicación en la inseminación sistemática de vacas secas y vaquillas cebú, Anales VI Simposio Internacional de Reproducción Animal, Córdoba (Argentina), p. 426.

12. SAS. 1989. SAS/STAT ${ }^{\circledR}$. User's Guide, Version 6, Fourth ed., vol. 2, Cary, NC; SAS Institute Inc., 846 p. 